\title{
Thermal Separation Library: Examples of Use
}

\author{
Karin Dietl ${ }^{*}$ Kilian Link ${ }^{\dagger}$ Gerhard Schmitz
}

\begin{abstract}
This paper deals with the Thermal Separation Library, which is intended to be used for absorption and rectification processes. Two example calculations show how the simulation speed can be increased by choosing the right way to set up the equations. One example refers to the ordering of the substances in the substance vector and one refers to the modelling of equilibrium processes. An example of use presented is the $\mathrm{CO}_{2}$ absorption in a post-combustion carbon capture plant. The transient simulation results are compared to measurement data obtained in a Siemens pilot plant.

Keywords: thermal separation, carbon capture, absorption / desorption
\end{abstract}

\section{Introduction}

Dynamic analysis of thermal separation processes gains in importance, be it in batch processing, system control, start-up strategies or shut down behaviour in continuous processing ([9]).

General modelling approaches for these problems (or a part of these problems) have been reported in literature. READYS ([16]) as well as a model developed in [12] can be used for dynamic simulation of equilibrium columns. A model which considers dynamic simulation of multiphase systems is presented in [2]. [11] describes steady-state and dynamic non-equilibrium models. A tool for dynamic process simulation - DIVA - is proposed by [5], which has a sequential simulation approach. DYNSIM was developed by [3] and is a tool for design and analysis of chemical processes. In [13] a modelling language gPROMS is proposed to model combined lumped and distributed systems which was then successfully used in several publications in order to model separation processes (e.g. [23]).

\footnotetext{
*Hamburg University of Technology, karin.dietl@tuhh.de

†Siemens, kilian.link@ siemens.com

†Hamburg University of Technology, schmitz@tuhh.de
}

The developed models have been tested on several different processes, mostly on a single column. There are also examples where more complex system designs are presented ([6]).

The aim of this paper is to describe a modelling library which can be used for flexible modelling and simulation of complex separation systems. As an example a fully integrated absorption/desorption loop for carbon capture is simulated. Simulation results will be compared to measurement data obtained in a Siemens pilot plant. The process is shown in figure 5 and will be discussed in more detail in section 4.1.

\section{Modelling Approach}

It is commonly agreed on that when developing a model of multicomponent system including chemical reactions, a simple equilibrium-based model which neglects mass transfer, is often not sufficient and a more physical approach - the rate-based approach ([18]) - is needed (e.g. [19], [17], [10]). The simulation models are built using the object-oriented Thermal Separation Library ([8]) which was developed in order to model dynamic absorption and rectification processes.

\subsection{Model equations}

\subsubsection{Balance equations}

The balance equations are established separately for the vapour and liquid bulk phase as described in [8]. There is therefore a mole balance for each component $i$ of vapour and liquid phase. Additionally there should also be summation equations in the bulk phases $\left(\sum_{i} y_{i}=1\right.$ and $\left.\sum_{i} x_{i}=1\right)$. However, in general systems of ordinary differential equations can be more easily solved than differential algebraic equations and introducing a differential equation instead of an algebraic equation is rewarded with faster computation times. Therefore these two algebraic equations are replaced by the total amount of substance balance for vapour and liquid phase.

For liquid and vapour phase there is one energy balance each (see [8]). 


\begin{tabular}{|llll|}
\hline \multicolumn{2}{|l|}{ Nomenclature } & \multicolumn{2}{l|}{ Greek symbols } \\
$A$ & heat transfer area & $\varepsilon^{\text {vap }, \varepsilon^{\text {liq }}}$ & vapour or liquid hold up \\
$a$ & specific area & {$[\Gamma]$} & thermodynamic correction matrix \\
$c$ & concentration & $\gamma$ & activity coefficient \\
$H$ & Henry coefficient & $\phi$ & fugacity coefficient \\
$k$ & mass transfer coefficient & Subscripts \\
$M$ & molar mass & $i$ & component $i$ \\
$\dot{N}$ & molar flow rate & $j$ & stage $j$ \\
$n$ & number of discrete elements & $r$ & reaction \\
$n_{S}$ & no. of substances which are in both phases & Superscripts \\
$n_{S}^{\text {liq }}, n_{S}^{\text {vap }}$ & number of liquid / vapour substances & liq & liquid \\
{$[R]$} & rate matrix for mass transfer coefficients & sat & saturation \\
$T$ & temperature & vap & vapour \\
$u$ & specific inner energy & $*$ & thermodynamic equilibrium \\
$x$ & liquid composition & Abbreviations \\
$y$ & vapour composition & nLF & number of liquid feeds \\
$Z$ & factor for equilibrium stage & nVF & number of vapour feeds \\
& & \multicolumn{2}{l}{} \\
\hline
\end{tabular}

\subsubsection{Rate Equations}

In [8] the molar flow rates at stage $j$ over the phase boundary are calculated as

$$
\overrightarrow{\dot{N}}_{i, j}=k_{i, j} \cdot a_{j} \cdot\left(\overrightarrow{c_{i, j}}-\overrightarrow{c_{i, j}^{*}}\right)
$$

This approach however is only valid for binary systems, and using the concentration difference as driving force is only applicable for isothermic conditions. Therefore this was replaced by the more general Maxwell-Stefan equations as described by [22]:

$\overrightarrow{\dot{N}}_{j}^{\mathrm{vap}}=\dot{N}_{\text {total }, j}^{\mathrm{vap}} \cdot \vec{y}_{j}+c_{j}^{\mathrm{vap}} \cdot a_{j} \cdot\left[R_{j}^{\mathrm{vap}}\right]^{-1} \cdot\left[\Gamma_{j}^{\mathrm{vap}}\right] \cdot\left(\overrightarrow{y_{j}}-\overrightarrow{y_{j}^{*}}\right)$

$\overrightarrow{\dot{N}}_{j}^{\mathrm{liq}}=\dot{N}_{t o t a l, j}^{\mathrm{liq}} \cdot \vec{x}_{j}+c_{j}^{\mathrm{liq}} \cdot a_{j} \cdot\left[R_{j}^{\mathrm{liq}}\right]^{-1} \cdot\left[\Gamma_{j}^{\mathrm{liq}}\right] \cdot\left(\vec{x}_{j}-\vec{x}_{j}^{*}\right)$

The thermodynamic correction matrices $[\Gamma]$ are necessary, since here the difference of the mole fraction as driving force is used and not the difference in the chemical potential. The $[\Gamma]$-matrices contain the composition derivatives of the activity coefficient (liquid) or fugacity coefficient (vapour), which can be found for several activity coefficent models in [21]. The $[R]$ matrices are calculated using the binary mass transfer coefficients. How to obtain the $[R]$-matrices via the binary mass transfer coefficients is described in detail in [22].

The total molar flow rates in (2) and (3) are obtained by summing the molar flow rates for each component.
It is also important to note that the vectores $\overrightarrow{\dot{N}}_{j}^{\text {vap }}$ and $\overrightarrow{\dot{N}}_{j}^{\text {liq }}$ in eq. (2) and (3) contain only the molar flow rate $\dot{N}_{j, i}$ of $n_{S}^{\text {vap }}-1$ and $n_{S}^{\text {liq }}-1$ substances respectively, that is this equation is not established for every substance.

The missing equations are the summation equations at the phase boundary:

$$
\sum_{i} y_{i}^{*}=1, \sum_{i} x_{i}^{*}=1
$$

\subsubsection{Inert Substances}

An equation describing the thermodynamic equilibrium does only exist for the $n_{S}$ components, which exist in both phases, but not for inert substances. The missing equations for the inert substances are obtained by setting the molar flow rate over the phase boundary of the inert substances to zero:

$$
\left.\begin{array}{c}
\dot{N}_{i, j}^{\mathrm{vap}}=0 \\
\dot{N}_{i, j}^{\mathrm{liq}}+\dot{N}_{r, i, j}^{\mathrm{film}}=0
\end{array}\right\} \text { if substance } i \text { is inert }
$$

\subsection{Numerical Solutions}

In order to solve the resulting system of differentialalgebraic equations using numerical integration, as many state variables as differential equations are needed. For the columns, the following variables are chosen as state variables: $c_{j, i}^{\text {vap }}, c_{j, i}^{\text {liq }}, u_{j}^{\text {vap }}, u_{j}^{\text {liq }}, \varepsilon_{j}^{\text {vap }}$ and $T_{j}^{\mathrm{vap}}$ (or $M_{j}^{\mathrm{vap}}$ which is as suitable), with $j=1 \ldots n$ and $i=1 \ldots n_{S}^{\text {vap }}$ or $i=1 \ldots n_{S}^{\text {liq }}$ respectively. Using this set 
of state variables, all other variables can be calculated via algebraic relations. Intensive rather than extensive quantities were chosen as state variables, since the differential equations were set up using these intensive variables.

\subsection{Library Structure}

The library structure of the column models is represented in figure 1 which shows a class diagram of the library. The three different column types (packed column, tray column, spray column) all inhert from FeedStage (which is denoted with an arrow pointing at the parent class). The FeedStage-model inherits from the BaseStage-model which contains the balance equations and some of the constitutive equations for $n$ discrete elements. It also contains instances of the medium models, the reaction models etc. These models are declared as replaceable, where replaceability is denoted using a dotted line with a diamond.

The extending column classes supply the geometry, the instances of the pressure loss and liquid holdup model, the heat transfer model between the two phases and the mass transfer models. Each column type is structured the same way; but only the structure of the packed column is shown in the diagram due to readability.

\section{Examples}

As stated in the introduction it should be possible to model and simulate a complex separation process using the Thermal Separation Library. In the following a complete absorption/desorption loop is presented. Before doing so some small examples show how a different writing of the modelling equations can increase simulation speed.

\subsection{Ordering of Substances in Medium Model}

In general any order of the substances in the medium model can be chosen. However since for a nonequilibrium model the equations for the molar flow rates eq. (2) and (3) exist only for $n_{S}^{\text {vap }}-1$ and $n_{S}^{\text {liq }}-1$ substances respectively, and the molar flow rate for the last substance in the medium model is defined via the summation equations (4), the ordering becomes important in case the fraction of the last substance becomes very small (in the order of the tolerance of the numerical solver). In this case the calculation can become very slow and the composition of the last sub- stance at phase boundary may even be calculated to be negative. This is known as cancellation problem (see e.g. [4]). Figure 2 shows an example for the absorption of $\mathrm{N}_{2}$ and $\mathrm{O}_{2}$ in $\mathrm{H}_{2} \mathrm{O}$. The simulation time decreases by the factor 5 if not $\mathrm{O}_{2}$ is the last substance in the medium model.

So it can be stated having substances with very low concentrations at the last place in the substance vector decreases simulation speed. If this is not possible (i.e. no substance is always large enough to be suitable as last component) it is also possible to apply the Maxwell-Stefan equations to all components and ommit the summation equations at the phase boundary. This also reduces the CPU-time, but in some test cases the sums of the mole fractions at the phase boundary were observed to deviate about $10 \%$ from 1 .

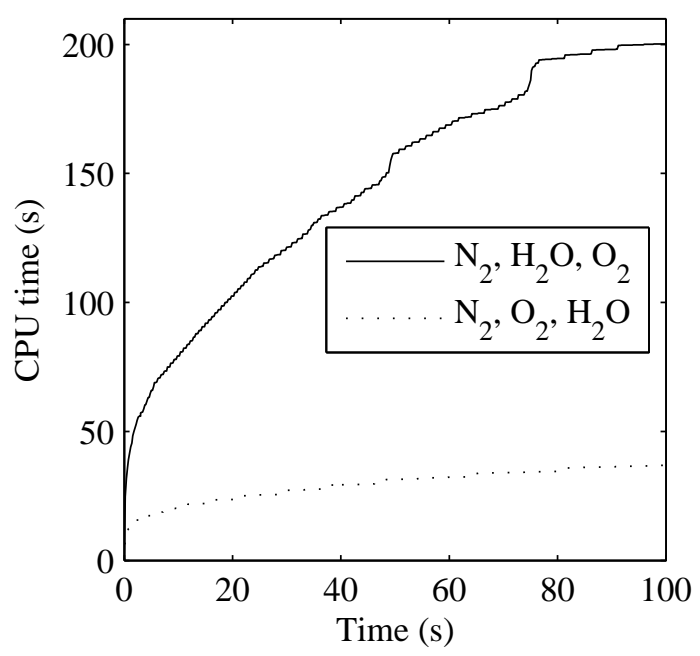

Figure 2: Influence of the ordering of the substances in the medium model. Simulation speed is increased, if in the liquid phase a substance with a high mole fraction $\left(\mathrm{H}_{2} \mathrm{O}\right)$ is the last substance in the medium model (i.e. the CPU time for simulation is smaller).

\subsection{Equilibrium Model}

As stated in chapter 2 the stages were modelled as nonequilibrium stages, i.e. mass transfer is taken into account. However sometimes it is advantageous to describe the separation column using equilibrium stages, e.g. if no suitable correlations for the mass transfer are available.

There are basically two different approaches to model such an equilibrium stage: The first possibility is to implement a new set of equations which describe the equilibrium stage. However the additional algebraic constraint of the compositions at phase boundary and 


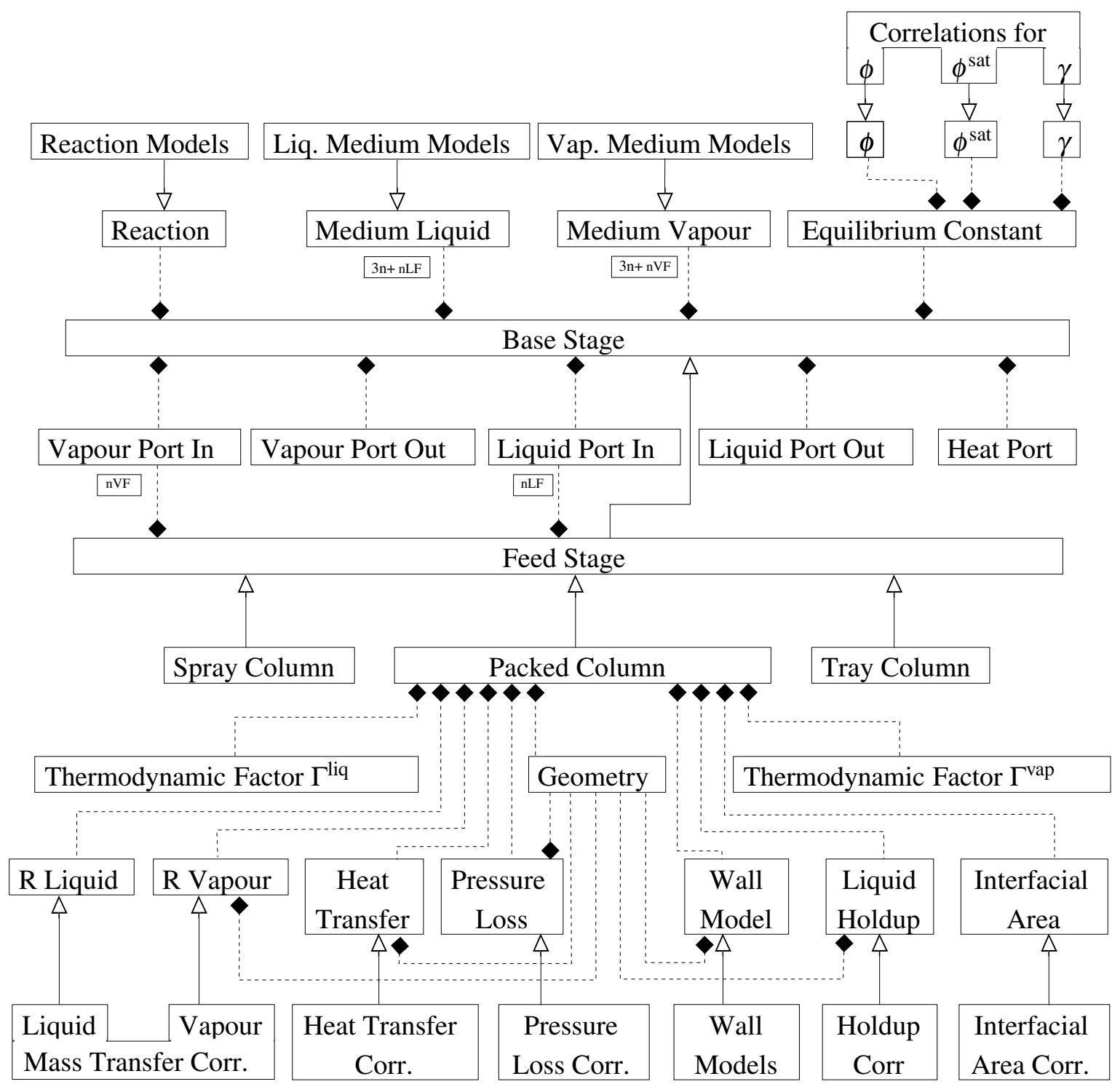

Figure 1: UML class diagram of an absorption or rectification process. The arrow denotes inheritance, i.e. FeedStage inherits from BaseStage only. The line with the diamond denotes composition. Dotted lines mean that the object is replaceable. The composition of spray column and tray column are analogue to the composition of the packed column. 
in the bulk phase being equal result in a large nonlinear system of equations.

The second possiblity is to approach the thermodynamic equilibrium $\left(x_{i} \rightarrow x_{i}^{*}\right)$ using the non-equilibrium equations and increasing the binary mass transfer coefficients to infinity. Using this approach the system is less strongly coupled, but still it has to be noted that the equations (2) to (4) introduce a high non-linearity to the system. Together with a very high value for the binary mass transfer coefficients, necessary in order to approach equilibrium, the computation time can become very large (see figure 3 ). Since the equation (2) - (4) do not give any information for the equilibrium model they are replaced by the much more simpler eq. (6)-(7):

$$
\begin{gathered}
\dot{N}_{j, i}^{\mathrm{vap}}=Z_{j, i}^{\mathrm{vap}} \cdot\left(y_{j, i}-y_{j, i}^{*}\right) \\
\dot{N}_{j, i}^{\mathrm{liq}}=Z_{j, i}^{\mathrm{liq}} \cdot\left(x_{j, i}-x_{j, i}^{*}\right)
\end{gathered}
$$

were $Z$ is an adjustable factor. If $Z$ approaches infinity, the difference between the composition at the phase boundary and in the bulk phase vanishes and equilibrium is attained. A very high value for $Z$ lead to a very accurate result. An indicator for the accuracy is the difference of the composition at the phase boundary to the composition in the bulk phase. This difference should become zero for an equilibrium model. However for a very high value for $Z$, the computation time may become very large or - even worse - there are convergence problems with the nonlinear solver. Since a optimal value for $Z$, which leads to an acceptable compromise between computing time and accuracy is not constant during simulation, $Z$ is continuously adapted to minimize the difference between bulk and phase boundary composition using the equations of a simple PI controller.

Equations (6) and (7) are then replaced by equations (8) and (9), where $Z$ are functions of the error $\left(y_{j, i}-\right.$ $\left.y_{j, i}^{*}\right)$ and $\left(x_{j, i}-x_{j, i}^{*}\right)$ respectively and some controller parameters which are constant during simulation.

$\dot{N}_{j, i}^{\mathrm{vap}}=Z_{j, i}^{\mathrm{vap}}\left(y_{j, i}-y_{j, i}^{*}\right.$, contr. parameter $) \cdot\left(y_{j, i}-y_{j, i}^{*}\right)$

$\dot{N}_{j, i}^{\mathrm{liq}}=Z_{j, i}^{\mathrm{liq}}\left(x_{j, i}-x_{j, i}^{*}\right.$, contr. parameter $) \cdot\left(x_{j, i}-x_{j, i}^{*}\right)$

Figures 3 and 4 show that using eq. (6) and (7) (solid line) or eq. (8) and (9) (dotted line) instead of eq. (2), (3) and (4) (dashed line) lead to a lower computation time and a higher accuracy of the result. Adapting the variable $Z$ during the simulation using a PI controller (dotted line) further increases accuracy

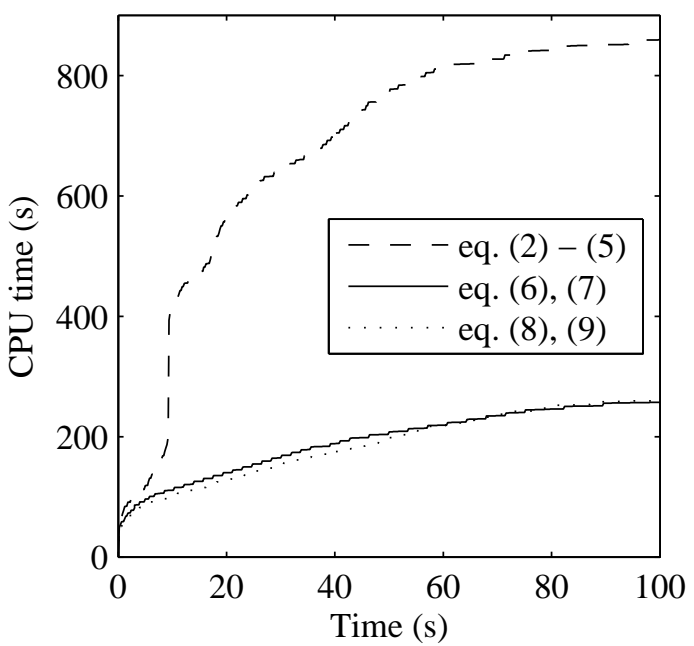

Figure 3: Computation time using three different sets of equations to model an equilibrium stage. The first set uses exactly the same equations as for a nonequilibrium model (eq. (2) to (4)) with a constant, but high mass transfer coefficient. The second and third set use simplified yet sufficient detailed equations which increases simulation speed.

without increasing the computation time, compared to eq. (6) and (7) where $Z$ is constant.

In this example, the first set of equations has more time states than the last two (178 scalars instead of 162 scalars; for eight stages) but about the same amount of time varying variables (around 12000). The size of the nonlinear system of equations obtained by Dymola is higher, for the first set of equations: eight blocks of 20 iterations variables are necessary instead of eight blocks of 15 iteration variables (the other blocks are identical with lesser iteration variables.

The same approach is also chosen when reaction equilibrium should be assumed: in this case the controller minimizes the difference between the reaction equilibrium constant and the product of the activities. 


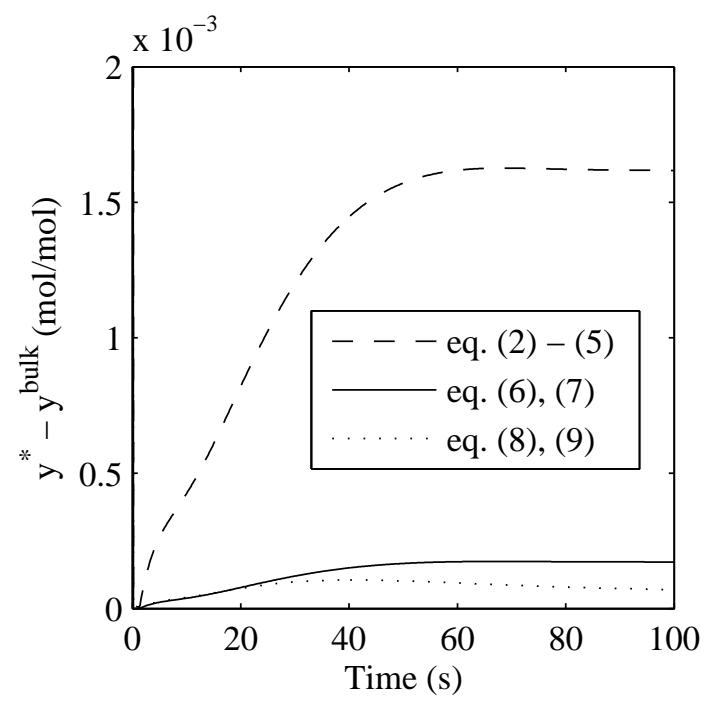

Figure 4: Largest difference in $y^{*}-y$ using three different sets of equations to model an equilibrium stage.

\section{Dynamic Analysis of $\mathrm{CO}_{2}$ Capture Plant}

\subsection{Plant Layout and Data}

The simulation of the carbon capture plant refers to the same pilot plant as presented in [7]. It is a slipstream pilot plant operating under real conditions. The absorber as a diameter of approx. DN200 and the absorber height is approx. $35 \mathrm{~m}$. The plant layout is shown in figure 5.

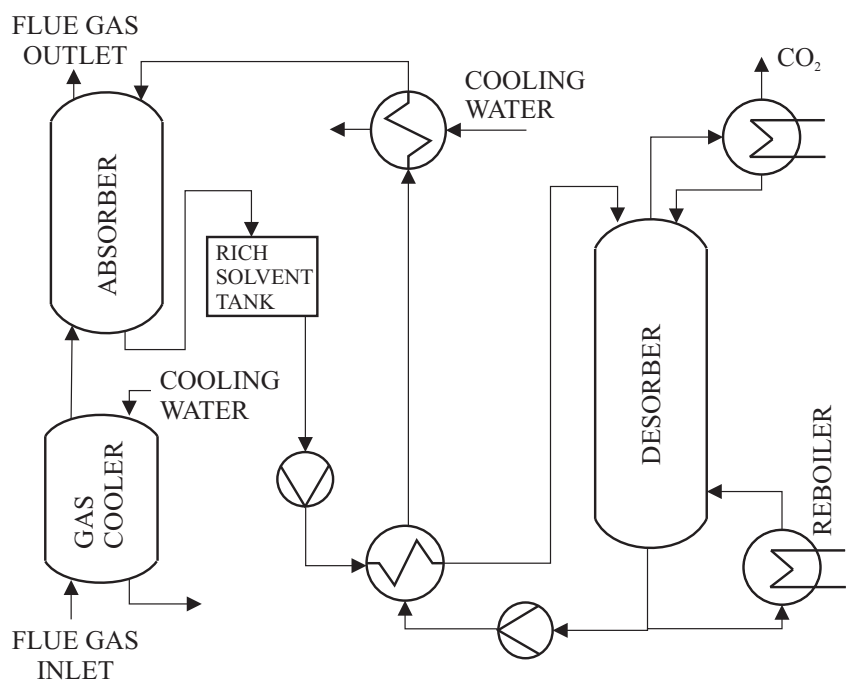

Figure 5: Carbon capture pilot plant as it can be found in [7].

The plant consists basically of a flue gas cooler, an absorber and a desorber (see figure 5). The flue gas first enters the flue gas cooler, where it is cooled down
Table 1: Physical properties and other parameters

\begin{tabular}{ll}
\hline Property & Reference \\
\hline Pressure loss vapour & {$[20]$} \\
Interfacial heat transfer coeff. & Chilton-Colburn \\
Mass transfer coefficient & {$[15]$} \\
Liquid holdup & {$[14]$} \\
Diffusion coeff. vapour & {$[1]$} \\
Interfacial area & {$[15]$} \\
\hline
\end{tabular}

using pure water. In the absorber, the saturated flue gas is then brought in contact with a liquid containing a high amount of dissolved amino acid salt. Here, the $\mathrm{CO}_{2}$ is absorbed by the liquid and nearly $\mathrm{CO}_{2}$-free gas leaves the absorber. The loaded liquid is now heated up in a heat exchanger before it enters the desorber, where the $\mathrm{CO}_{2}$ is stripped from the liquid using water vapour, which is obtained in a thermosyphon. The gas leaving the desorber (containing water and $\mathrm{CO}_{2}$ ) is cooled down and such water is removed via condensation. The pure $\mathrm{CO}_{2}$ is then liquified and stored; the pure water is fed back to the desorber. The now unloaded liquid from the desorber bottom is partly evaporated in a thermosyphon and partly led back to the absorber via two heat exchangers in order to cool down to the absorber temperature.

The solvent used is an amino-acid salt solution, as described in [7]. The underlying chemical reaction is shown in figure 6. In the model, it is assumed that reaction takes place in the film only and reaction kinetics are not considered.

Table 1 provides an overview of the most important physical properties.

\subsection{Thermodynamic equilibrium}

\subsubsection{Phase equilibrium $\mathrm{CO}_{2}$}

A very simple approach proposed by Siemens was choosen to model the phase equilibrium of $\mathrm{CO}_{2}$ between liquid and gas phase. This approach combines the dissolution of $\mathrm{CO}_{2}$ in the liquid and the stepwise reaction with the dissolved salt to the final product in a single phase equilibrium equation. The main advantage is that only very few components in the liquid phase have to be modelled, namely $\mathrm{H}_{2} \mathrm{O}$, the dissolved salt called AAS (amino acid salt) and the final reaction product. All reaction intermediate products as well as dissolved molecular $\mathrm{CO}_{2}$ in the liquid phase have not to be considered, the latter due to the 


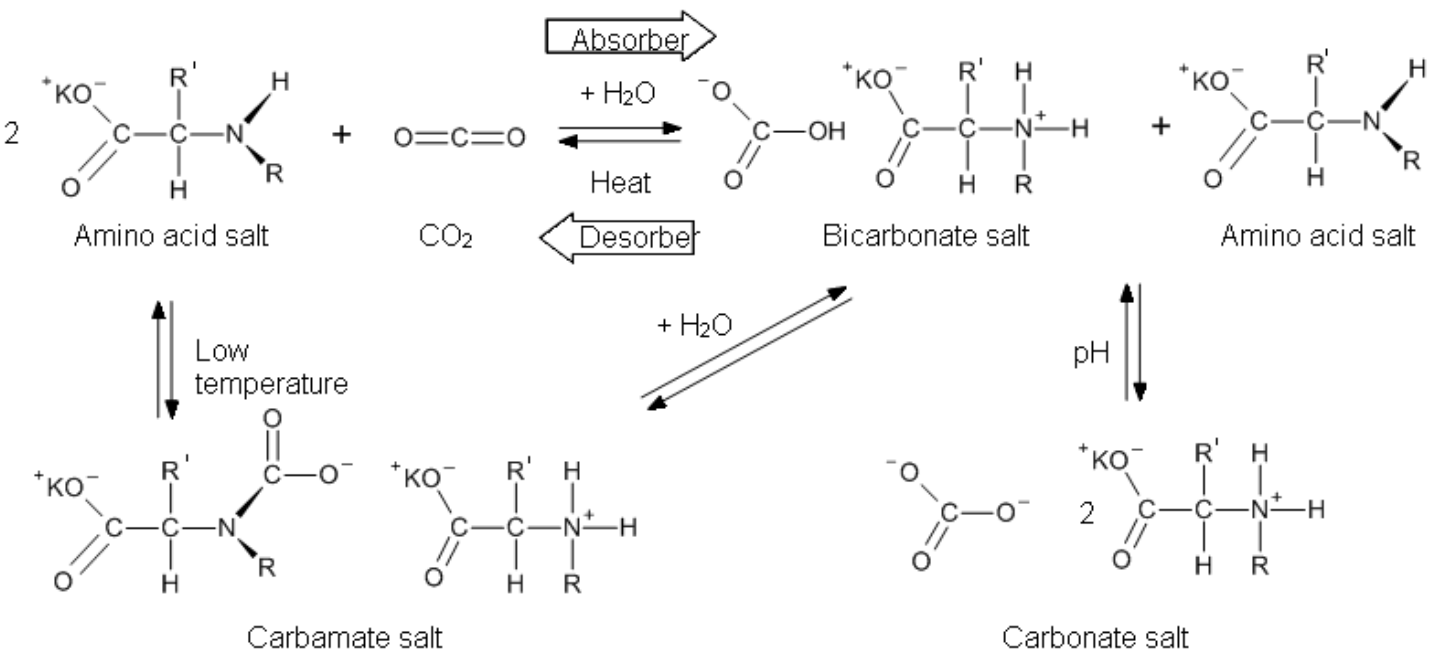

Figure 6: Reaction scheme in the amino acid salt solution, as presented in [7]

assumption that the reaction is nearly instantenous and the $\mathrm{CO}_{2}$ concentration in the liquid is negligible. This means that if a molar flow rate $\dot{N}_{\mathrm{CO}_{2}}$ of gaseous $\mathrm{CO}_{2}$ crosses the phase boundary, a source term increases the molar quantity of the reaction product and a sink term decreases the molar quantity of the educts $\left(\mathrm{H}_{2} \mathrm{O}\right.$, AAS) such accounting for the stoichiometrics.

Since $\mathrm{CO}_{2}$ is nonexistent in the model of the liquid phase the phase equilibrium is described using the $\mathrm{CO}_{2}$ vapour mole fraction on the one side and the mole fraction of the reaction product on the other side:

$$
p \cdot y_{\mathrm{CO}_{2}}=\frac{x_{\text {Product }}}{1+x_{\text {Product }}} \cdot H(T) \cdot \gamma\left(T, x_{\text {Product }}\right)
$$

The Henry coefficient $H$ is temperature dependent and the activity coefficient $\gamma$ is additionally dependent on the concentration of the product in solvent. For both coefficients Siemens provides a correlation, which are as follows:

$$
\begin{gathered}
\ln H=a+\frac{b}{T} \\
\ln \gamma=e^{\left(c+d \cdot T+e \cdot T^{2}\right)} \cdot \frac{x_{\text {Product }}}{1+x_{\text {Product }}}
\end{gathered}
$$

The parameters $a, b, c, d, e$ have been adjusted using measurement data.

It has to be stated that this approach has drawbacks concerning the accurateness of the results, nevertheless they are all in the right order of magnitude and the main factors influencing the result are considered. This approach is therefore suitable for dynamic simulation, where the dynamics are in the focus of the study and a high simulation speed is needed but very correct steady-state results are not of such importance.

\subsubsection{Phase equilibrium water}

The water equilibrium is calculated using

$$
p \cdot y_{\mathrm{H}_{2} \mathrm{O}}=x_{\mathrm{H}_{2} \mathrm{O}} \cdot p^{\mathrm{sat}} \cdot \gamma \cdot \phi^{\mathrm{sat}}
$$

For the calculation of the water saturation pressure, the saturation pressure of pure water is multiplied by a factor smaller 1 to match the higher saturation temperature. The activity coefficient $\gamma$ is set equal to one, even though it should be smaller than one in reality. At Siemens steady-state calculations were performed using AspenPlus where much more detailed medium models than the ones here where used. These AspenPlus calculations revealed that the activity coefficient did not differ significantly from one in the considered temperature and concentration range. Therefore, since the proposed modelling of the thermodynamic equilibrium is anyway not suitable in order to obtain very accurate steady-state results, setting the water activity coefficient equal to one does not increase the overall error very much.

\subsection{Medium models}

\subsubsection{Gas mediums}

Both gases, the flue gas, consisting of $\mathrm{N}_{2}, \mathrm{CO}_{2}, \mathrm{O}_{2}$ and $\mathrm{H}_{2} \mathrm{O}$ and the $\mathrm{CO}_{2}$-water vapour mixture in the desorber, are modelled as ideal gas, even though this assumption is more questionable for the $\mathrm{CO}_{2}$-water vapour mixture, due to the high water vapour content (about 70\%-90\%) at the saturation temperature of water (around $100^{\circ} \mathrm{C}$ ). However the error due to differences in density and enthalpy are not important. 


\subsubsection{Liquid mediums}

The pure water in the flue gas cooler is modelled using the waterIF97 medium model from the Modelica Standard Library.

The solvent is also modelled based on the water IF97 standard, but density and heat capacity are adjusted to match the solvent values (for a solvent containing approx. 30 weight $-\%$ AAS). All solvent medium properties are temperature and in parts also pressure dependent, but independent of composition.

As stated in 4.2.2 the solvent model consists only of three components in order to increase simulation speed.

\subsection{Simulation results}

In this section the simulation results are presented and compared to measurement data obtained in the Siemens pilot plant.

Two different test cases are investigated:

- Test case 1: Increase of Flue gas flow rate from $75 \%$ to $100 \%$ at $\mathrm{t}=0 \mathrm{~min}$.

- Test case 2: Decrease of steam flow rate at reboiler from $90 \%$ to $75 \%$ at $\mathrm{t}=0 \mathrm{~min}$.

Figure 7 - 9 show the comparision between simulation and measurements of test case 1 . All temperatures are plotted referring to an arbitrary reference temperature. The $\mathrm{CO}_{2}$ mass flow rate is described in percent, whereas $100 \%$ is arbitrarily set to the measurement value obtained at $\mathrm{t}=0 \mathrm{~min}$. It can be seen that the steady-state results differ, which is due to the very simplified modelling of the thermodynamic equilibrium. The dynamic response between simulation and measurement are quite similar, even though in the simulation the new steady-state is obtained after a shorter time.

For test case 2 the comparision between simulation and measurements are shown in figure $10-14$. Again, all temperatures are plotted referring to an arbitrary reference temperature. As for test case 1 the steadystate results differ, but the dynamic behaviour is similar. In figure 12 not only the measured $\mathrm{CO}_{2}$ mass flow rate at the desorber outlet is plotted, but also the mass flow rate of the absorbed $\mathrm{CO}_{2}$ in the absorber. The latter value was obtained from measurement data, using measured mass flow rates, temperatures and volume fractions at the absorber in- and outlet. These two mass flow rates should be equal in steady-state (which

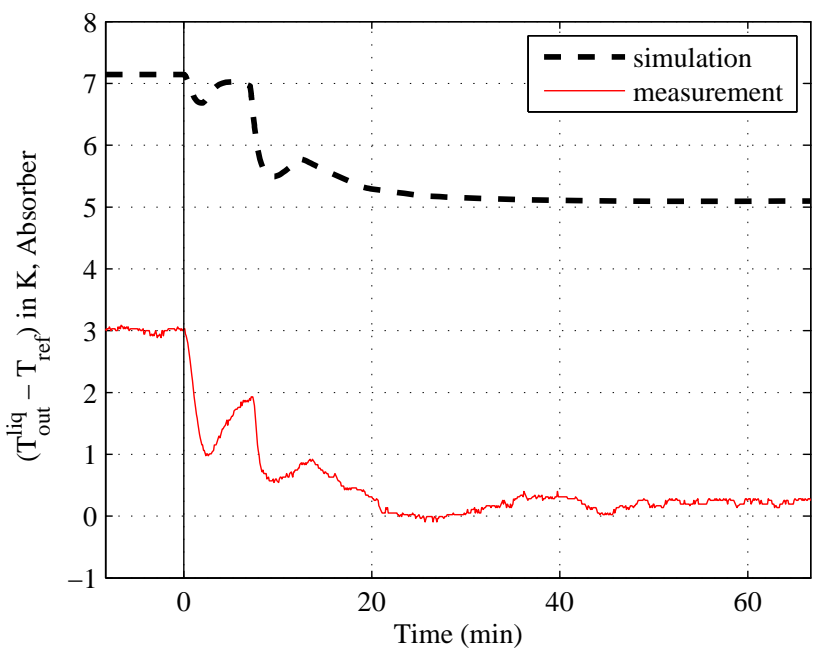

Figure 7: Liquid outlet temp., absorber (test case 1)

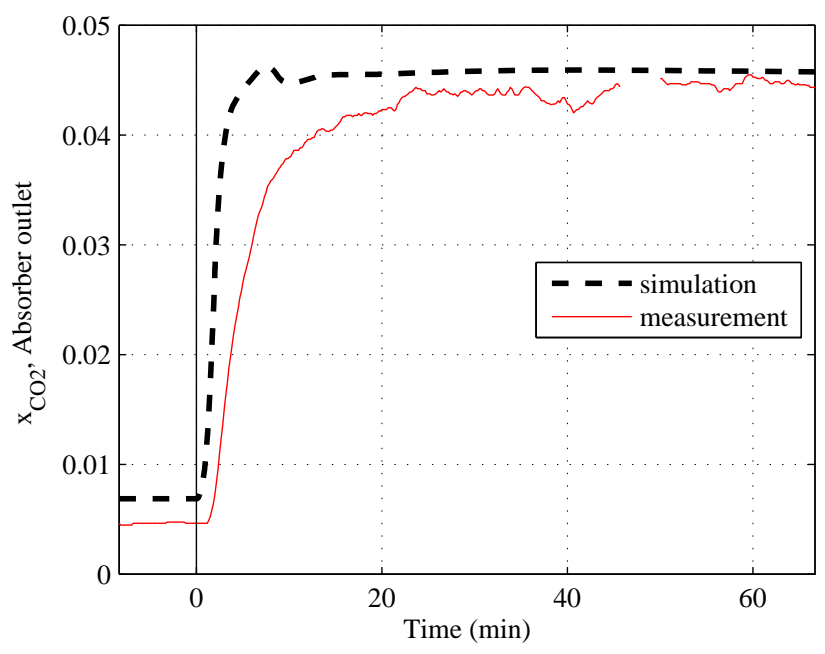

Figure 8: $\mathrm{CO}_{2}$ outlet mole fraction (test case 1)

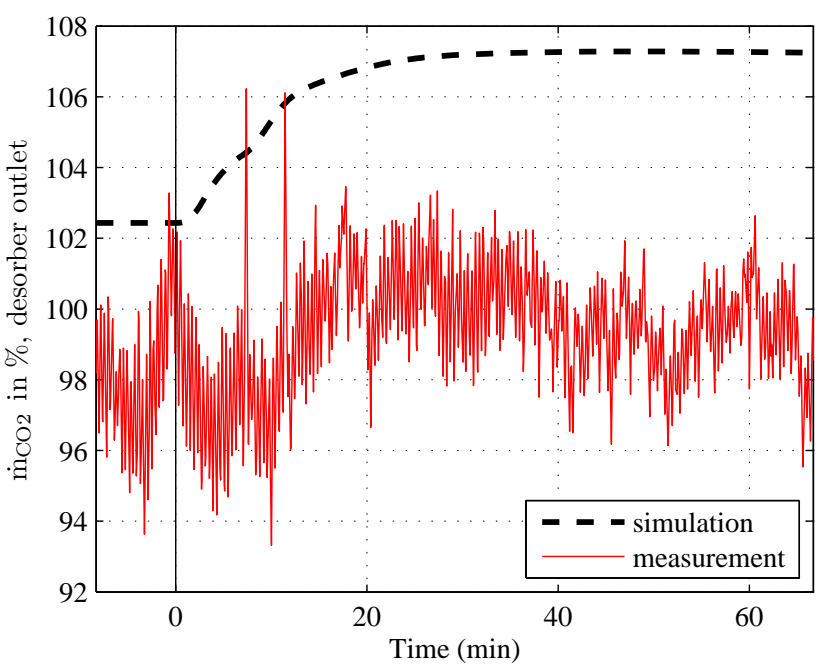

Figure 9: $\mathrm{CO}_{2}$ mass flow rate, desorber (test case 1) 


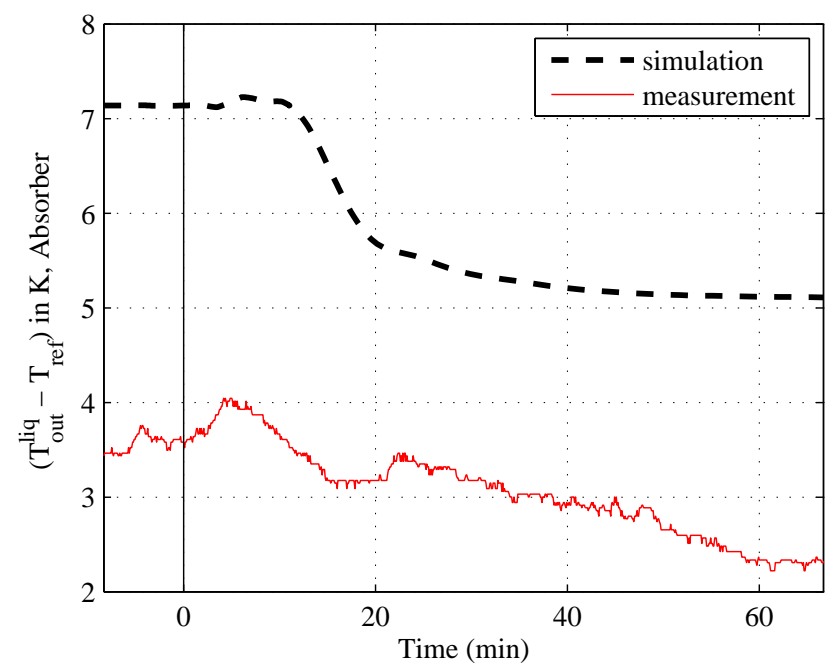

Figure 10: Liquid outlet temp., absorber (test case 2)

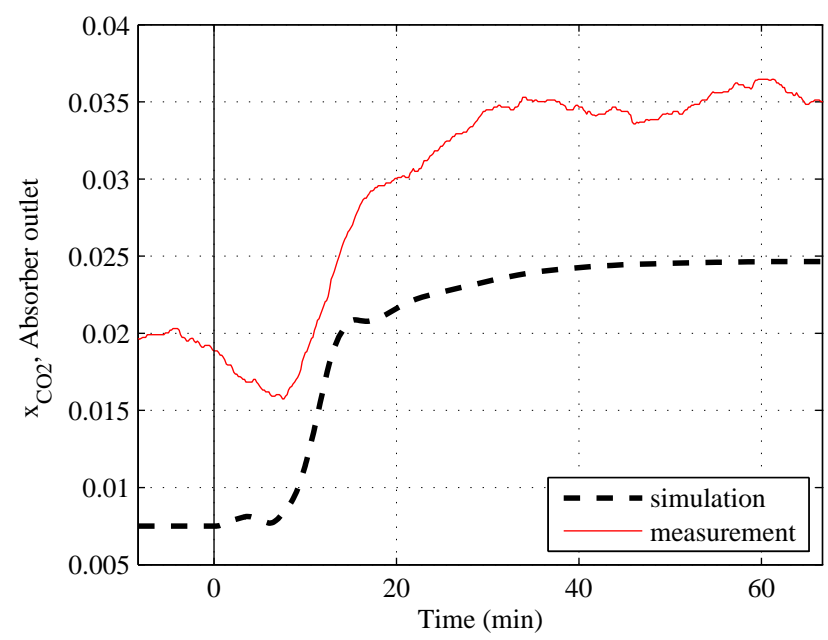

Figure 11: $\mathrm{CO}_{2}$ outlet mole fraction (test case 2)

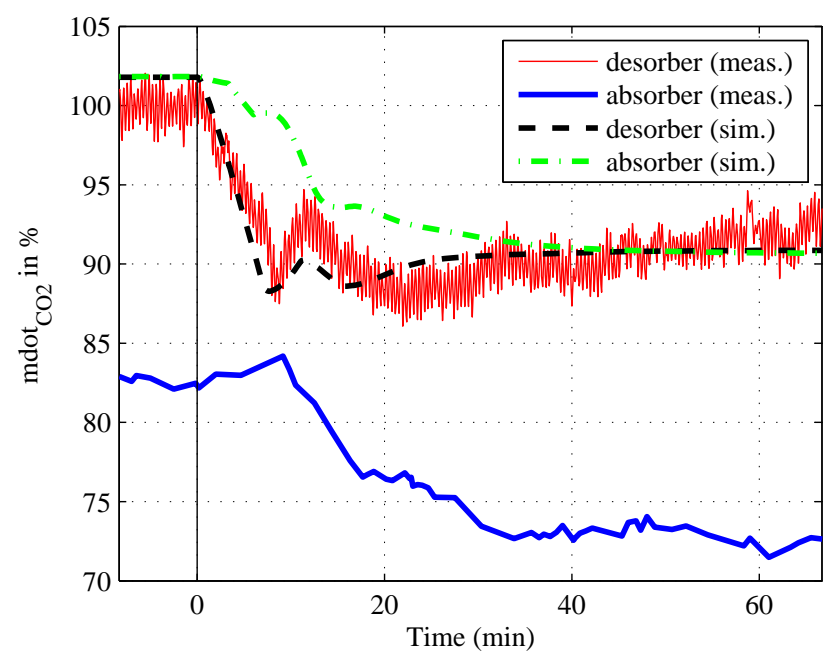

Figure 12: $\mathrm{CO}_{2}$ mass flow rate (test case 2) was the case in test case 1), however there is a difference of about $20 \%$. Since this difference does not occur in the simulation, it explaines why measurement and simulation fit well for the $\mathrm{CO}_{2}$ mass flow rate at the desorber outlet, but not for the $\mathrm{CO}_{2}$ mole fractions in the absorber and consequently not for the amount of $\mathrm{CO}_{2}$ absorbed.

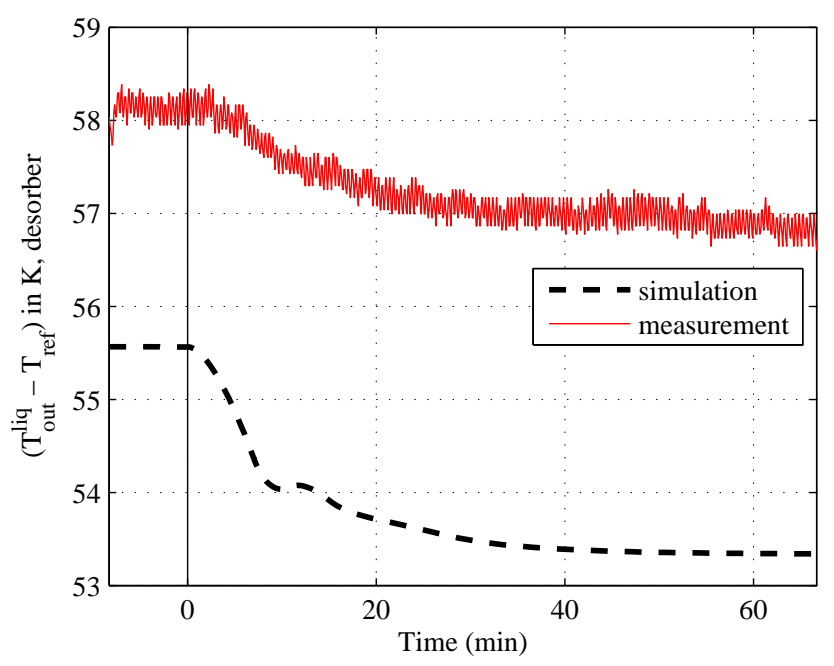

Figure 13: Liquid outlet temp., desorber (test case 2)

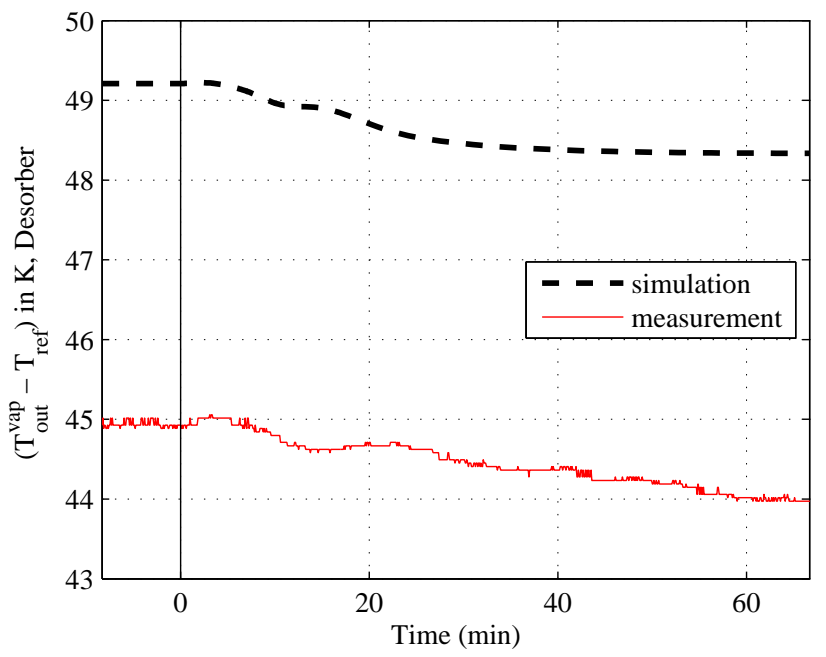

Figure 14: Vapour outlet temp., desorber (test case 2)

\section{Summary}

This work proposed a Modelica-library model for dynamic simulation of tray and packed columns for separations processes such as absorption and rectification. It is shown that it is advantageous to describe an equilibrium model using modified equations of the nonequilibrium model. Also the influence of the ordering 
of the substances in the medium vector was shown. It was also shown that using the Thermal Separation Library a complex system, namely an absorption/desorption loop for carbon capture is modelled and simulated dynamically. The simulation results were compared to measurement data obtained in a Siemens pilot plant. It showed good agreement, even though there were differences in the stationary results which is due to the very simplified modelling of the thermodynamic equilibrium.

\section{References}

[1] Edward N. Fuller, Paul D. Schettler, and J. Calvin Giddings. A new method for predicition of binary gas-phase diffusion coefficients. Industrial and Engineering Chemistry, 58(5):19-27, May 1966.

[2] R. Gani, Thomas S. Jespen, and Eduardo S. Perez-Cisneros. A generalized reactive separation unit model. modelling and simulation aspects. Computers Chemical Engineering, 22(Supplement):363-370, 1998.

[3] R. Gani, Esben L. Sorensen, and Jens Perregaard. Design and analysis of chemical processes through DYNSIM. Ind. Eng. Chem. Res., 31:244-254, 1992.

[4] David Goldberg. What every computer scientist should know about floating-point arithmetic. ACM Computing Surveys, 23(1):5-48, March 1991.

[5] P. Holl, W. Marquardt, and E. D. Gilles. DIVA a powerful tool for dynamic process simulation. Computers chem. Engng, 12(5):421-426, 1988.

[6] Bernhard Hüpen and E. Kenig. Rigorose modellierung und simulation von chemisorptionsprozessen. Chemie Ingenieur Technik, 77(11):1792-1798, 2005.

[7] Tobias Jockenhoevel, Ruediger Schneider, and Helmut Rode. Development of an economic post-combustion carbon capture process. Energy Procedia, 1:1043-1050, 2009.

[8] Andreas Joos, Karin Dietl, and Gerhard Schmitz. Thermal separation: An approach for a modelica library for absorption, adsorption and rectification. In Francesco Casella, editor, Proceedings of the 7th International Modelica Confer- ence, Linköping Electronic Conference Proceedings, pages 804-813. Linköping University Electronic Press, September 2009.

[9] E. Kenig. Complementary modelling of fluid separation process. Chemical Engineering Research and Design, 86:1059-1072, 2008.

[10] E. Kenig, Kaj Jakobsson, Peter Banik, Juhani Aittamaa, and Andrzej Gorak. An integrated tool for synthesis and design of reactive distillation. Chemical Engineering Science, 54:1347-1352, 1999.

[11] Hendrik A. Kooijman. Dynamic Nonequilibrium Column Simulation. PhD thesis, Clarkson University, 1995.

[12] J. M. Le Lann, J. Albet, X. Joulia, and B. Koehret. A multipurpose dynamic simulation system for multicomponent distillation columns. Computer Applications in Chemical Engineering, pages 355-359, 1990.

[13] M. Oh and Constantinos C. Pantelides. A modelling and simulation language for combinend lumped and distributed parameter systems. Computers chem. Engng, 20(6/7):611-633, 1996.

[14] J. Antonio Rocha, J. L. Bravo, and J. R. Fair. Distillation columns containing structured packings: A comprehensive model for their performance 1 . hydraulic models. Ind. Eng. Chem. Res., 32:641$651,1993$.

[15] J. Antonio Rocha, J. L. Bravo, and J. R. Fair. Distillation columns containing structured packings: A comprehensive model for their performance. 2. mass transfer model. Ind. Eng. Chem. Res., 35:1660-1667, 1996.

[16] C. A. Ruiz, M. S. Basualdo, and N. J. Scenna. Reactive distillation dynamic simulation. Institution of Chemical Engineers, pages 363-378, 1995.

[17] M. Schenk, R. Gani, D. Bogle, and E. N. Pistikopoulos. A hybrid modelling approach for separation systems involving distillation. Trans IChemE, 77:519-534, 1999.

[18] J. D. Seader. The rate-based approach for modeling staged separation. Chemical Engineering Progress, pages 41-49, 1989. 
[19] M. S. Sivasubramanian and Joseph F. Boston. The heat and mass transfer rate-based approach for modeling multicomponent separation processes. Computer Applications in Chemical Engineering, pages 331-336, 1990.

[20] J. Stichlmair, J. L. Bravo, and J. R. Fair. General model for predicition or pressure drop and capacity of countercurrent gas/liquid packed columns. Gas Separation \& Purification, 3:19-28, March 1989.

[21] Ross Taylor and Hendrik A. Kooijman. Composition derivatives of activity coefficient models. Chem. Eng. Comm., 102:87-106, 1991.

[22] Ross Taylor and R. Krishna. Multicomponent mass transfer. John Wiley \& Sons, Inc., 1993.

[23] M. L. Winkel, L. C. Zullo, P. J. T. Verheijen, and Constantinos C. Pantelides. Modelling and simulation of the operation of an industrial batch plant using gPROMS. Computers chem. Engng, 19:571-576, 1995. 